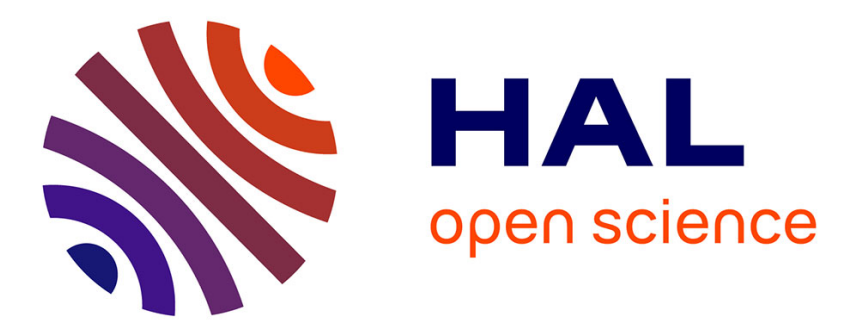

\title{
Development of MHD flow in the entrance region of a channel
}

\author{
S.T. Wu, T.S. Fu, M.B. Wear
}

\section{To cite this version:}

S.T. Wu, T.S. Fu, M.B. Wear. Development of MHD flow in the entrance region of a channel. Revue de Physique Appliquée, 1971, 6 (3), pp.409-414. 10.1051/rphysap:0197100603040900 . jpa-00243563

\section{HAL Id: jpa-00243563 https://hal.science/jpa-00243563}

Submitted on 1 Jan 1971

HAL is a multi-disciplinary open access archive for the deposit and dissemination of scientific research documents, whether they are published or not. The documents may come from teaching and research institutions in France or abroad, or from public or private research centers.
L'archive ouverte pluridisciplinaire HAL, est destinée au dépôt et à la diffusion de documents scientifiques de niveau recherche, publiés ou non, émanant des établissements d'enseignement et de recherche français ou étrangers, des laboratoires publics ou privés. 


\title{
DEVELOPMENT OF MHD FLOW IN THE ENTRANCE REGION OF A CHANNEL
}

\author{
S. T. WU \\ University of Alabama in Huntsville \\ T. S. FU \\ Mechanical Sciences Lab., Brown Engineering Huntsville, Alabama \\ and M. B. WEAR \\ University of Alabama in Huntsville \\ (Reçu le 8 mai 1970, révisé le 3 mai 1971)
}

\begin{abstract}
Résumé. - Une méthode générale a été développée pour déterminer le flux magnétohydrodynamique se développant à l'entrée d'un canal à plaques parallèles. Une forme fermée pour les solutions a été obtenue. Les résultats numériques sont présentés pour le développement de la distribution des vitesses, la chute de pression et la longueur de l'entrée magnétohydrodynamique pour différents nombres de Hartman. Pour de grandes valeurs du nombre de Hartman, l'effet de champ magnétique devient prédominant et la longueur d'entrée magnétohydrodynamique devient négligeable. D'autre part, la chute de pression est accrue par le champ magnétique. Les résultats sont en parfait accord avec ceux obtenus par une analyse numérique directe.

Abstract. - A general method has been developed for determining the developing magnetohydrodynamic flow in the entrance region of a parallel-plate channel. Closed form solutions are obtained. Numerical results are presented for the development of velocity distribution, pressure drop, and magnetohydrodynamic entrance length at different Hartmann numbers. At large Hartmann numbers, the magnetic field effect becomes dominant and the magnetohydrodynamic entrance length becomes negligible. Also, the pressure drop is enhanced by the magnetic field. Comparison of results with direct numerical analysis shows excellent agreement.
\end{abstract}

Introduction. - The study of developing flow in the entrance region of a parallel-plate channel for a conducting fluid has become increasingly important in the design of magnetohydrodynamic accelerators, generators, induction flow meters and similar devices. As is well-known, for a non-conducting fluid, the velocity distribution of the flow will undergo a development from an initial profile at the inlet to a fullydeveloped profile far downstream and, correspondingly, the pressure gradient in the region of flow development will differ from that of a fully developed flow. When the fluid becomes conducting, a similar problem of magnetohydrodynamic flow needs to be solved. Different methods have been used by a number of investigators [1] in an attempt to obtain solutions for the MHD flow problem by using a velocity profile matching procedure after Schilchting's approximate method [2]. The same problem has also been solved using a numerical method of the finite difference analysis by Shohet, Osterle and Young [3], and by

(*) Work done by STW and MBW was supported by NASA Grant NGL 01-002-001.
Hwang and Fan [4]. Recently, Sparrow, Lin and Lundgren [5] proposed a new method to determine the developing laminar flow and corresponding pressure drop in the entrance region of tubes and ducts for a non-conducting fluid. In their method, a linearized procedure is employed to obtain the velocity distribution along the length from the channel entrance to the fully developed region.

In the present study, we have applied the method proposed by Sparrow, Lin and Lundgren to a laminar incompressible magnetohydrodynamic flow in a parallel-plate channel with non-conducting channel walls. The velocity profile at the entrance region is obtained in a closed form and the fully developed velocity profile is of Hartmann's flow [6]. The expression for the pressure drop is also derived. The purpose of the present study is to show that the method proposed by Sparrow et al. [5] can be applied to the case of an electrically conducting fluid and the numerical calculations easily performed. Finally, the results have been presented for the velocity distribution, pressure drop, and magnetohydrodynamic entrance length (i. e., the length of the channel in which the velocity development occurs) at different Hartmann numbers. 
Basic Equations. - Consideration is given to a laminar incompressible flow of electrically conducting fluid with a uniformly applied transverse magnetic field, $H_{0}$ in a parallel-plate channel for small magnetic Reynolds number flow. Hence, the magnetic field will be independent of the fluid motion. The channel axis is taken to lie along the positive $x$-direction, while $y$ and $z$ are the cross-sectional coordinates (e. g., Fig. 1). The equations governing the problem as described previously may be written as:

$$
\begin{gathered}
\frac{\partial u}{\partial x}+\frac{\partial v}{\partial y}=0 \\
U \frac{\partial u}{\partial x}+v \frac{\partial u}{\partial y}+\left(\frac{M}{h}\right)^{2} v u=-\frac{1}{\rho} \frac{\partial \omega}{\partial x}+v \frac{\partial^{2} u}{\partial y^{2}}
\end{gathered}
$$

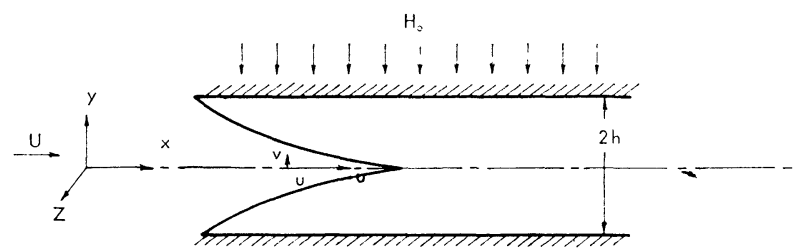

Fig. 1. - Geometry of parallel plate channel at entrance region with transverse magnetic field and uniform entrance velocity.

with

$$
M=\mu H_{0} h\left(\frac{\sigma}{v \rho}\right)^{1 / 2},
$$

the Hartmann Number, and

$$
\omega=p-\left(\frac{M}{h}\right)^{2} \rho v \frac{E x}{\mu H_{0}}
$$

in which $u(x, y)$ and $v(x, y)$ are the velocity components in the $x$ and $y$ directions, respectively. The other symbols are the pressure $p$, the density $\rho$, the kinematic viscosity $v$, the electric conductivity $\sigma$, the external applied magnetic field $H_{0}$ along the $y$-direction and finally, the magnetic permeability $\mu$, and the $E$ being the electric field strength normal to the magnetic and flow field directions. However, the second term in $(2 a)$ can be neglected by assuming that the length normal to the $x-y$ plane is long enough, and this could be done by connecting two conducting plates with a low resistance wire ; therefore, the $E$ will become insignificant. Hence, eq. (2) becomes

$$
U \frac{\partial u}{\partial x}+v \frac{\partial u}{\partial y}+\left(\frac{M}{h}\right)^{2} v u=-\frac{1}{\rho} \frac{\partial p}{\partial x}+v \frac{\partial^{2} u}{\partial y^{2}} .
$$

Eq. (3) represents the conservation of momentum along the $x-y$ direction. In deriving this equation, the boundary layer type assumptions have been used. The eq. (1) represents conservation of mass.

(*) The authors are indebted to referee for this comment.
Velocity Solutions. - In the present analysis, the velocity can be expressed as a sum

$$
u=u_{f \mathrm{~d}}+u^{*}
$$

in which $u_{f \mathrm{~d}}$ is the fully-developed velocity distribution and $u^{*}$ is a difference velocity. $u^{*}$ will be of significance only in the entrance region and will approach zero at large downstream distance. As is well known, the fully-developed velocity solution for the present problem is that of Hartmann's solution which may be written as

$$
u_{f \mathrm{~d}}=\frac{1-\cosh M y / \cosh M}{1-\tanh M / M} .
$$

To determine the velocity solution in the entrance region, we seek solutions of the following modified linearized momentum equation after Sparrow et al. [5] ;

$$
\varepsilon(x) U \frac{\partial u}{\partial x}+\frac{v M^{2}}{h^{2}} u=\Lambda(x)+v \frac{\partial^{2} u}{\partial y^{2}},
$$

where $\varepsilon(x)$ is a yet undetermined function of $x$ which weights, $U$, the mean velocity. $\Lambda(x)$ is another undetermined function which can be obtained by integrating eq. (5) over the heights of the channel noting that

$$
\int_{-h}^{h}\left(\frac{\partial u}{\partial x}\right) d y=0
$$

to satisfy mass conservation. This gives

$$
\Lambda(x)=\frac{v M^{2} U}{h^{2}}-\left.\frac{v}{h} \frac{\partial u}{\partial y}\right|_{y=h} .
$$

On the right hand side of eq. (6) ; the first term represents magnetic field effects and the second term represents effects due to pressure gradient as well as the viscous term. Substituting eq. (6) into (5), yields

$\varepsilon(x) U \frac{\partial u}{\partial x}+\frac{v M^{2}}{h^{2}} u=\frac{v M^{2} U}{h^{2}}-\left.\frac{v}{h} \cdot \frac{\partial u}{\partial y}\right|_{y=h}+v \frac{\partial^{2} u}{\partial y^{2}}$.

Because of the linearity property of eq. (7), it can be shown by substituting eq. (4) into eq. (7)

$\varepsilon(x) U \frac{\partial u^{*}}{\partial x}+\frac{v M^{2}}{h^{2}} u^{*}=-\left.\frac{v}{h} \cdot \frac{\partial u^{*}}{\partial y}\right|_{y=h}+v \frac{\partial^{2} u^{*}}{\partial y^{2}}$.

Introducing the following dimensionless parameters

$$
\left.\begin{array}{rl}
u^{*} & =U u^{* \prime}, \\
y & =h y^{\prime}, \\
x & =\frac{U h^{2}}{v} x^{\prime} .
\end{array}\right\}
$$


Eq. (8) becomes, after dropping the superscript prime for all variables,

$$
\varepsilon(x) \frac{\partial u^{*}}{\partial x}+M^{2} u^{*}=\frac{\partial^{2} u^{*}}{\partial y^{2}}-\left.\frac{\partial u^{*}}{\partial y}\right|_{y=1}
$$

A solution for $u^{*}$ can be found from eq. (10) by introducing a stretched axial coordinate $x^{*}$, which may be defined

$$
\mathrm{d} x=\varepsilon \mathrm{d} x^{*}
$$

Thus

$$
u^{*}=\sum_{i} c_{i} g_{i}(y) \mathrm{e}^{-\alpha_{i}^{2} x}
$$

wherein $g_{i}$ satisfies

$$
\frac{\mathrm{d}^{2} g_{i}}{\mathrm{~d} y^{2}}+\beta_{i}^{2} g_{i}=g_{i}^{\prime}(1)
$$

with the boundary conditions

$$
g_{i}( \pm 1)=0
$$

where

$$
\beta_{i}^{2}=\alpha_{i}^{2}-M^{2} .
$$

Following the method outlined by Sparrow et al. [5], the solution for $g_{i}$ can be derived from eq. (13) as

$$
g_{i}(y)=\frac{\cos \beta_{i} y}{\cos \beta_{i}}-1,
$$

and

$$
c_{i}=\frac{2}{\alpha_{i}^{2}},
$$

wherein the eigenvalues, $\beta_{i}$ are the roots of

$$
\tan \beta_{i}=\beta_{i},
$$

and $\alpha_{i}$ will be determined by eq. (13c). The first 25 values for $\beta_{i}$ have been computed by following the method of Sparrow et al.

The velocity solution is then constructed by summing $u^{*}$ and $u_{f \mathrm{~d}}$.

$$
\begin{aligned}
& u=\frac{1-\cosh M y / \cosh M}{1-\tanh M / M}+ \\
& \quad+\sum_{i=1}^{\infty} \frac{2}{\alpha_{i}^{2}}\left(\frac{\cos \beta_{i} y}{\cos \beta_{i}}-1\right) \mathrm{e}^{-\alpha_{i}^{2} x^{*}} .
\end{aligned}
$$

The velocity solution is not completed until the relationship between the stretched coordinate $x^{*}$ and the physical coordinate $x$ is provided. This can be done by evaluating the stretching factor $\varepsilon(x)$ from eq. (3). With some mathematical manipulation, one obtains

$$
\varepsilon=\frac{\int_{0}^{1}\left(2 u-1.5 u^{2}\right) \frac{\partial u}{\partial x^{*}} \mathrm{~d} y}{\int_{0}^{1} \frac{\partial u^{2}}{\partial y} \mathrm{~d} y+M^{2}\left(\int_{0}^{1} u^{2} \mathrm{~d} y-1\right)+u^{\prime}(1)} .
$$

From the nature of the velocity solution, it is seen that integrations of eq. (18) can only be carried out numerically.

The relationship between $x$ and $x^{*}$ can now be determined easily by carrying out the integration of eq. (11) by knowing $\varepsilon$. Thus, with the relationship between $x$ and $x^{*}$ available, the velocity solution for the parallel-plate channel is complete.

Pressure Drop. - To determine the pressure drop in the entrance region, one may integrate eq. (3) over the cross-sectional area. Thus the pressure drop between the channel inlet (pressure $p_{0}$ ) and any axial location (pressure $p$ ) can be expressed in the following form :

$$
\begin{aligned}
& \frac{p_{0}-p}{\frac{1}{2} \rho U^{2}}=2\left(\int_{0}^{1} u^{2} \mathrm{~d} y-1\right)+ \\
& \quad+2\left(M^{2} x-\left.\int_{0}^{x^{*}} \frac{\partial u}{\partial y}\right|_{y=1} \varepsilon \mathrm{d} x^{*}\right) .
\end{aligned}
$$

It is convenient to rewrite this equation as

$$
\begin{aligned}
& \frac{p_{0}-p}{\frac{1}{2} \rho U^{2}}=\left[-\left.2 \frac{\partial u_{f \mathrm{~d}}}{\partial y}\right|_{y=1}\right] x+ \\
& \quad+2\left(\int_{0}^{1} u^{2} \mathrm{~d} y-1\right) \\
& \quad+2\left(M^{2} x-\left.\int_{0}^{x^{*}} \frac{\partial\left(u-u_{f \mathrm{~d}}\right)}{\partial y}\right|_{y=1} \varepsilon \mathrm{d} x^{*}\right) .
\end{aligned}
$$

Similarly to the expression obtained by Sparrow et al., the first term on the right still can be identified as the pressure drop which would be sustained by a flow if it were fully developed from the channel inlet and can be evaluated directly from the fully developed velocity solution as

$$
\left[-\left.2 \frac{\partial u_{f \mathrm{~d}}}{\partial y}\right|_{y=1}\right] x=f x
$$

in which $f$ is the fully developed friction factor and in the present case is given by

$$
f=\frac{2 M \tanh M}{M-\tanh M} .
$$

One may note that this friction factor is now a function of the magnetic field and the viscosity.

The other terms appearing on the right side of eq. (19a) represents the increment in pressure drop due to the development of the flow, and may be written as

$$
\begin{aligned}
K(x) & =2\left(\int_{0}^{1} u^{2} \mathrm{~d} y-1\right)- \\
& -\left.2 \int_{0}^{x^{*}} \frac{\partial}{\partial y}\left(u-u_{f \mathrm{~d}}\right)\right|_{y=1} \varepsilon \mathrm{d} x^{*}+2 M^{2} x .
\end{aligned}
$$


This expression reveals the same features as the work of Sparrow et al. On the right side, the first term represents a change in momentum between the entrance section $(x=0)$ and the down stream section, the second term represents the effects of shear stress on the wall by the developing flow relative to that of a fully developed flow, and the third term represents the direct effects due to the magnetic field. The indirect effect of the magnetic field is coupled in the first and second term through the velocity solution. On inspection of the expression for $K$, we notice that as the flow approaches its fully developed state, $K$ varies linearly with $M^{2}$ as in Hartmann's flow. Thus, eq. $(19 a)$ becomes

$$
\frac{p_{0}-p}{\frac{1}{2} \rho U^{2}}=f x+K(x)
$$

which retains the same form as obtained by Hwang and Fan, and Sparrow et al.

Results and Discussions. - The velocity profile for $M$ (Hartmann number) of 0,4 , and 10 in the developing and the fully developed region is shown in figure 2 for various $x$. As expected, the velocity

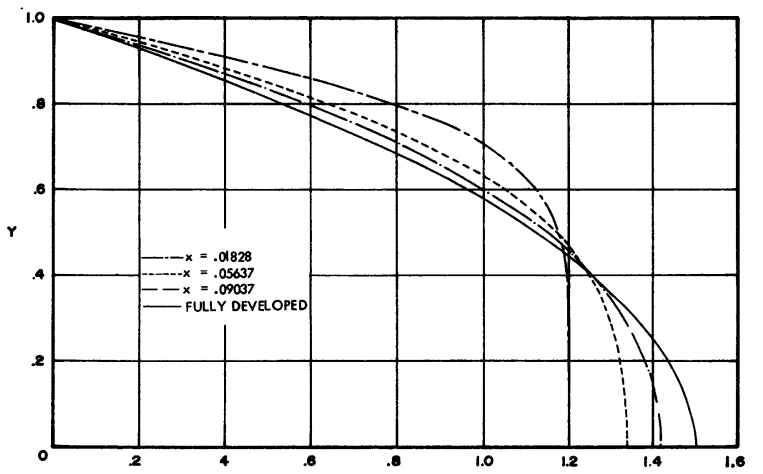

FIG. $2 a$. - Development of velocity profiles in a parallel-plate channel, $M=0$.

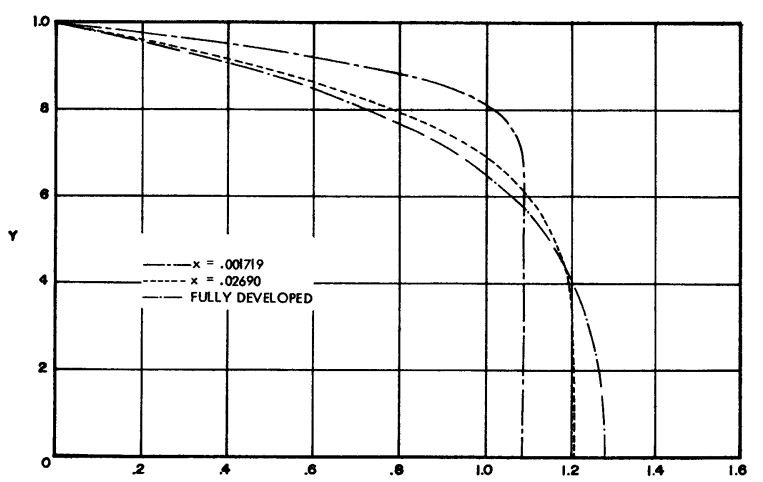

FIG. $2 b$. - Development of velocity profiles in a parallel-plate channel, $M=4$.

profile approaches a parabola with a dimensionless center line velocity similar to the results obtained by Hwang and Fan [4]. The profiles in the immediate neighborhood of the entrance are seen to have a

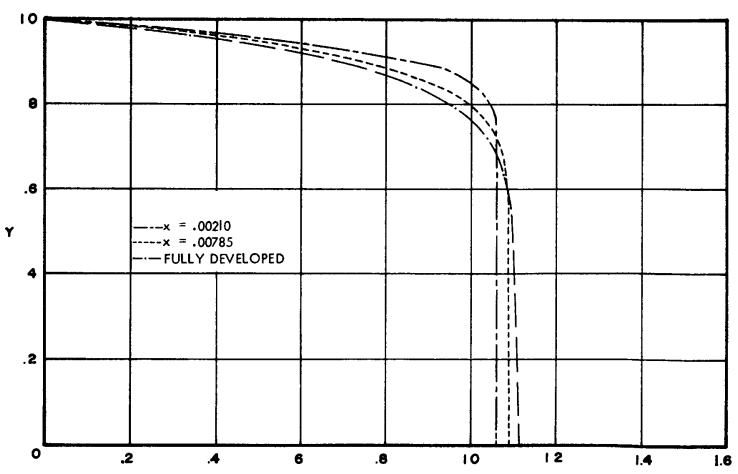

FIG. $2 c$. - Development of velocity profiles in a parallel-plate channel, $M=10$.

distinct flat portion and the magnetic field enhances this effect which leads to enhancement of the developing flow. Also, the axial development of the velocity profile for $M$ (Hartmann number) of 0,4 , and 10 in the entrance region is shown in figures $3 a, 3 b$ and $3 c$ for various values of $y$.

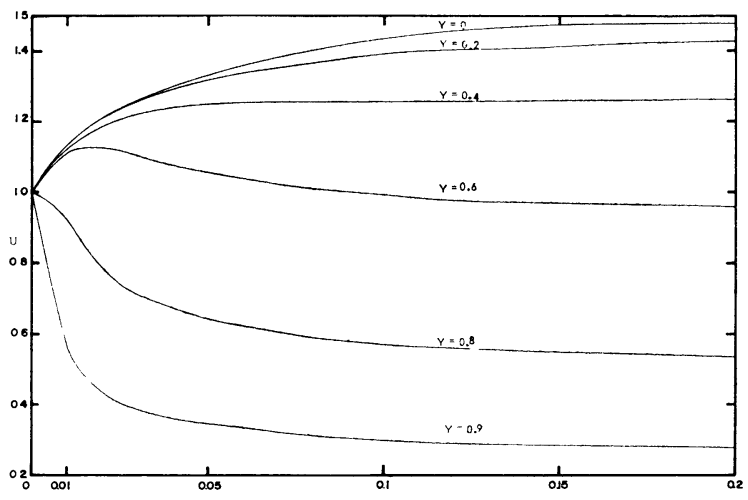

FIG. 3a. - Axial development of the velocity in a parallel-plate channel, $M=0$.

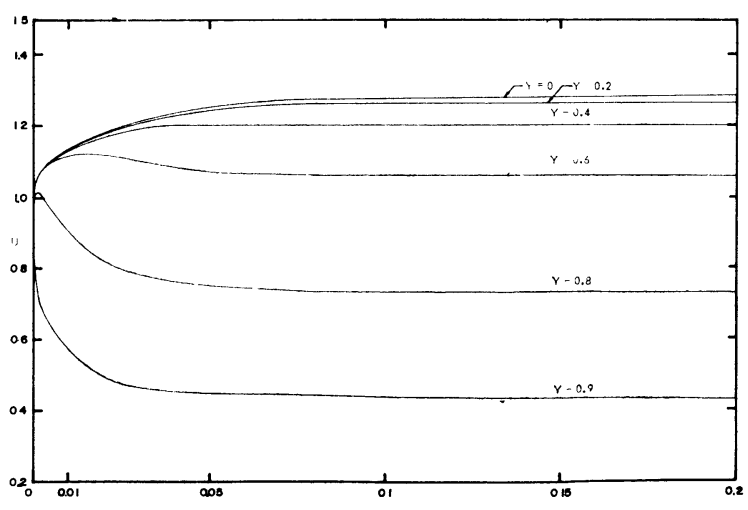

FIG. 3b. - Axial development of the velocity in a parallel-plate channel, $M=4$.

Table I shows the entrance length for one per cent and five per cent deviation of center line velocity from fully developed flow. The results are compared with those obtained by Schlichting [2], Roidt and Cess [1], and Hwang and Fan [4] for $M=0,4$, and 


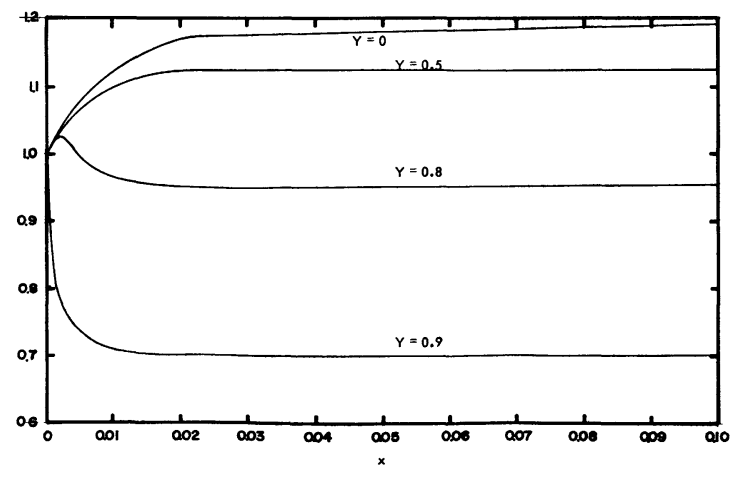

Fig. 3c. - Axial development of the velocity in a parallel-plate channel, $M=10$.

10. The results shown from the present analysis agree very well with those of Hwang and Fan, but there is a considerable difference with the results of Roidt and Cess.

TABLE I

\section{Comparison of the Entrance Length}

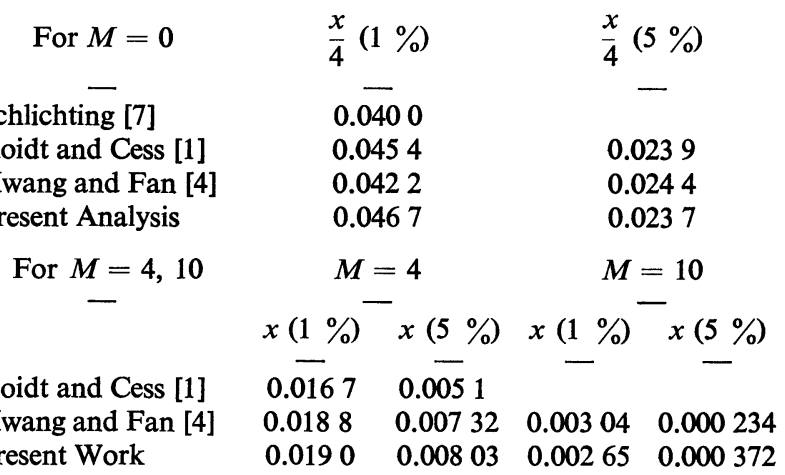

The relationship between the $x$ and $x^{*}$ is determined by carrying out the integration of $\varepsilon$ as indicated in eq. (18). This information is plotted in figure 4 at

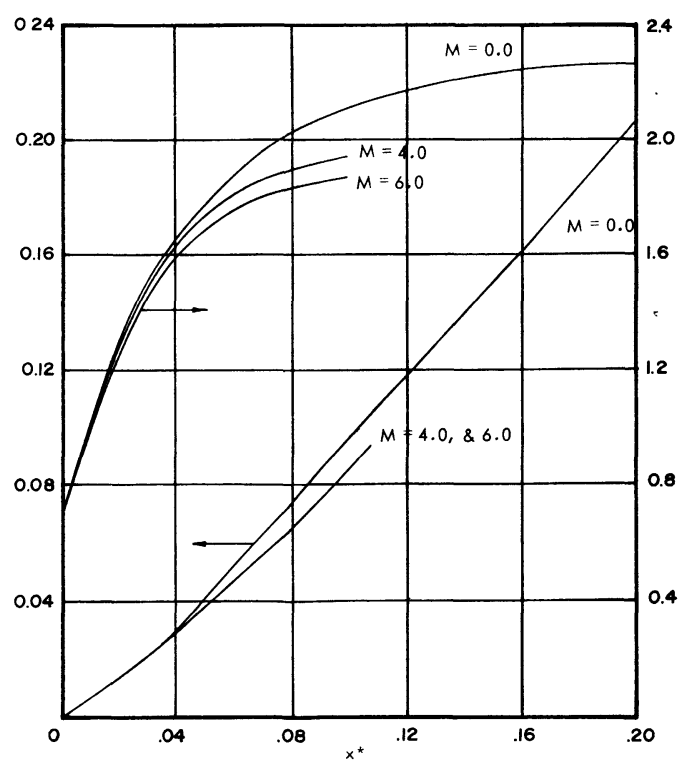

FIG. 4. - Relationship between the physical coordinate $x$ and the stretch coordinate $x^{*}$, parallel-plate channel, at $M=0,4$ and 6 . various Hartmann numbers in which the $x$ appears on the left-hand ordinate and $x^{*}$ on the abscissa. As is seen at $M=0$, this is identical with the results of Sparrow et al. The entrance length vs. Hartmann number is plotted in figure 5 .

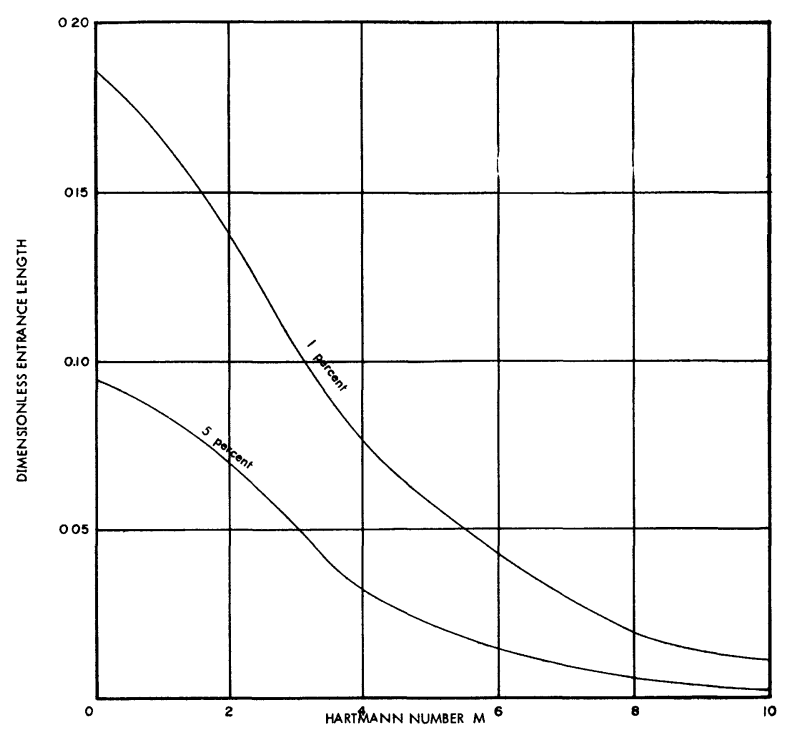

FIG. 5. - Entrance length vs. Hartmann number $M$.

Figure 6 shows the dimensionless pressure drops over the entrance region for various values of $\boldsymbol{M}$. These show good agreement with the results of Hwang and Fan.

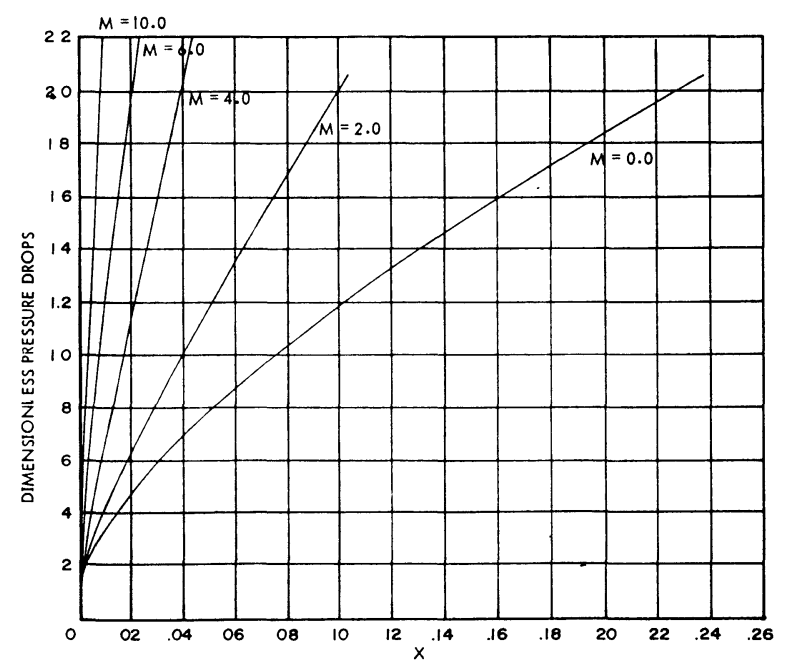

FIG. 6. - Dimensionless entrance-length pressure distribution at different Hartmann number.

In conclusion, the present analytical analysis has shown some improvement over the results of Roidt and Cess by employing Schlichting's method and is very close to the numerical analysis given by Hwang and Fan. We have not included the electric field in the present study because we limit ourselves to the 
case of solid electrodes and continuous electrical conductors. Thus, in essence, it will short-circuit out any Hall field that might be generated ; and the charge separation induced Hall effect is always several order of magnitudes smaller than the force field which we are interested. However, this analysis will not be affected by the inclusion of an electric field because this case only adds a constant term in our momentum equations. Therefore, the results for an electrically conducting channel wall can be easily obtained.

The authors are indebted to Prof. F. C. Todd for reading the manuscript and making suggestions.

\section{References}

[1] Roidt (M.) and CeSs (R. D.), Trans. of ASME, J. of Applied Mechanics, 1962, 171-176.

[2] Schlichting (H.), Boundary Layer Theory, 4th Ed., McGraw-Hill Book Co., New York, 1960, 169-171.

[3] Shohet (J. L.), Osterle (J. F.) and Young (F. J.), Phys. of Fluids, 1962, 5, 545-549.
[4] Hwang (C. L.) and FAn (L. T.), Appl. Sci. Res., B 10, 1963, 329-343.

[5] Sparrow (E. M.), Lin (S. H.) and Lundgren (T. S.), Phys. of Fluids, 1964, 7, 338-347.

[6] Cowling (T. G.), Magnetohydrodynamics, Interscience Publishers LTD, London, 1958, 13-15. 